International Journal of Case Reports
(ISSN:2572-8776)

\title{
Colo-renal fistula following percutaneous radiofrequency ablation of a renal tumor. A case report and review of treatment options
}

\author{
Allan Stolarski, $M D^{1,2}$, Joanna Wang, $M D^{1,2}$, Katherine He, $M D^{1,3}$, Lori Lerner, $M D^{1,2}$, Gentian \\ Kristo, MD $^{1,3}$ \\ ${ }^{1}$ Department of Surgery, Veterans Affairs Boston Healthcare System, Boston, MA, USA; \\ ${ }^{2}$ Department of Surgery, Boston Medical Center, Boston University Medical School, Boston, MA, \\ USA; ${ }^{3}$ Department of Surgery, Brigham and Women's Hospital, Harvard Medical School, Boston, \\ MA, USA.
}

\section{ABSTRACT}

Background: Colo-renal fistula formation is a very rare complication following percutaneous radiofrequency ablation (RFA) in the management of renal cell carcinoma. In this paper we describe the management of a patient with a left colo-renal fistula occurring after renal tumor RFA. Additionally, we present a thorough literature review of reported cases of colo-renal fistulae after percutaneous RFA to further highlight their treatment challenges.

*Correspondence to Author: Gentian Kristo, MD, MPH, FACS

Department of Surgery, Veterans Affairs Boston Healthcare System (112-C), 1400 VFW Parkway, West Roxbury, MA, 02132, USA Tel: +1857-2013-6966 Fax: +1-857-2035738 E-mail: gentian.kristo@va.gov

Summary: Two weeks after undergoing percutaneous RFA of an incidental $3 \mathrm{~cm}$ left renal mass, a 67-year-old male was re-admitted to the hospital with a symptomatic colo-renal fistula. PaHow to cite this article: tient ultimately failed conservative management with broad spectrum antibiotics and ureteral stent placements, and subsequently underwent segmental colonic resection and renorrhaphy, with complete renal preservation.

Allan Stolarski, Joanna Wang, Katherine He, Lori Lerner, Gentian Kristo. Colo-renal fistula following percutaneous radiofrequency ablation of a renal tumor. A case report Conclusion: Although complications after RFA of renal tumors are rare, iatrogenic colo-renal fistula formation is a significant complication requiring a multi-disciplinary approach with a trial of non-operative management prior to surgical intervention.

Keywords: renal cancer; radiofrequency ablation, colo-renal fistula and review of treatment options. International Journal of Case Reports, 2020 4:139

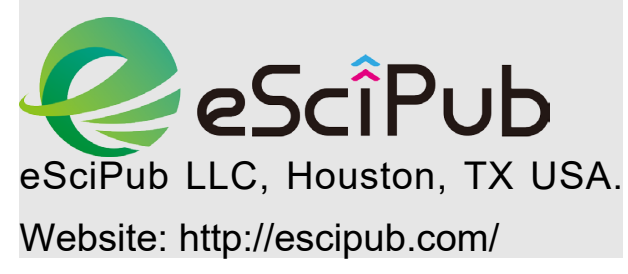




\section{Introduction:}

Radiofrequency ablation (RFA) of renal tumors is a minimally-invasive technique that plays an increasingly important role in the treatment of renal cancer. However, RFA of kidney lesions puts the surrounding organs and tissues at risk for thermal injury. latrogenic colo-renal fistula is a very rare complication of percutaneous RFA of renal cell carcinoma, and only a few cases have been described in the literature ${ }^{1-5}$.

In this article, we report on a patient with a left colo-renal fistula after RFA of a renal mass who failed conservative management and required an open takedown of the fistula with kidney salvage. The reported treatment options of colo-renal fistulae are also presented.

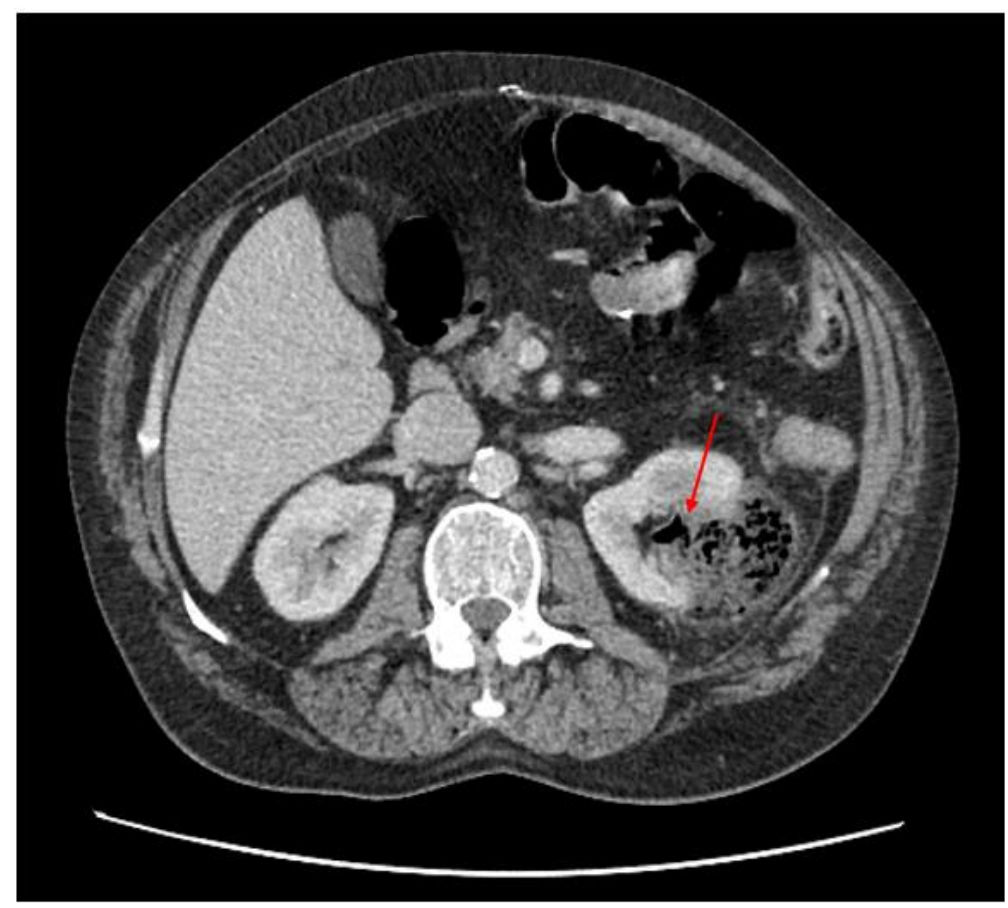

Figure 1. Axial CT scan two weeks after left renal RFA showing the area of ablation contiguous to the splenic flexure of the colon, as well as air within the collecting system of the left kidney suggesting a colo-renal fistula (arrow).

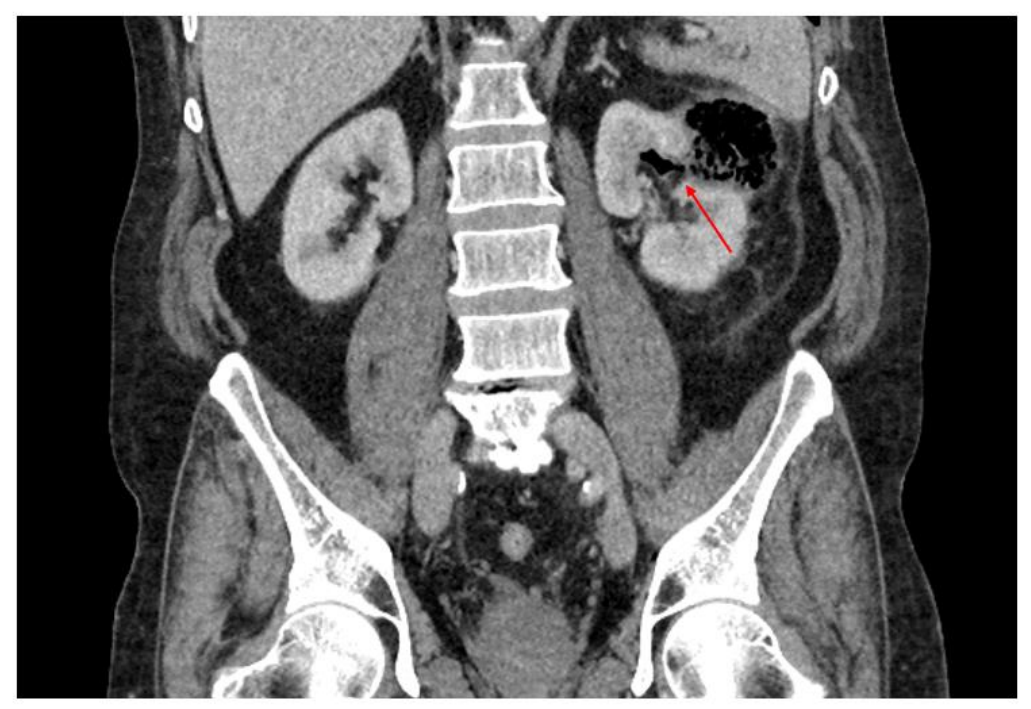

Figure 2. Coronal CT scan image showing a fistula formation between the splenic flexure of the colon and the upper pole of the left renal calyx (arrow). 


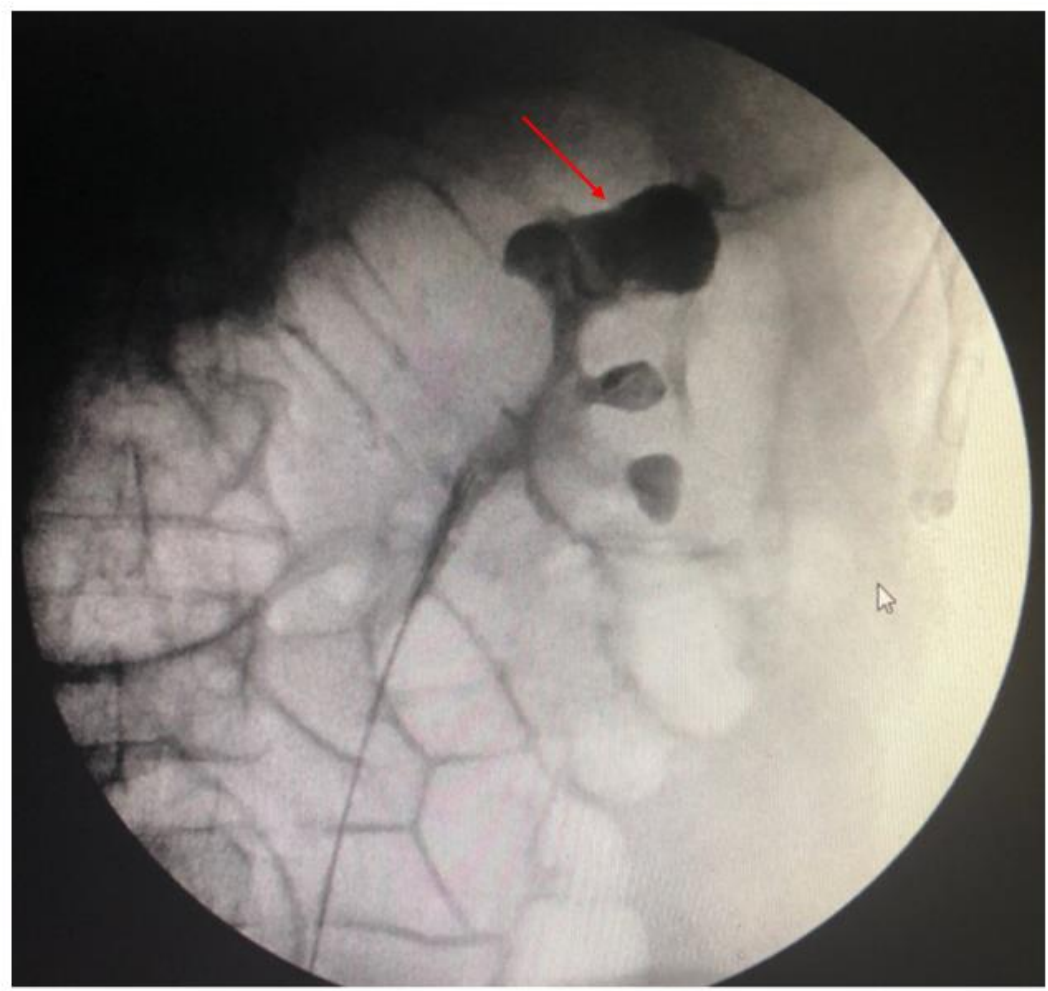

Figure 3. Left retrograde pyelogram confirming a colo-renal fistula by showing extravasation of contrast from the left upper pole calyx into the splenic flexure of the colon (arrow).

\section{Case Description}

A 67-year-old man who was found to have an incidental $3 \mathrm{~cm}$ left renal mass underwent a percutaneous computer tomography (CT)-guided biopsy and RFA by Interventional Radiology. Biopsy results showed clear cell renal carcinoma (Fuhrman Grade I-II). Two weeks after the procedure, the patient was re-admitted to the hospital with fever, dizziness, myalgias, left flank pain, pneumaturia, fecaluria, and profuse diarrhea with urine content. CT imaging revealed a left renal abscess with gas inside the calyceal system (Figure 1) and a fistula between the splenic flexure of the colon and the upper pole of the left renal calyx (Figure 2). The patient was started on broad-spectrum antibiotics, and 48 hours later underwent a cystoscopy and left retrograde pyelogram that showed extravasation of contrast from the left upper pole calyx into the splenic flexure of the colon (Figure 3). As placement of a stent into the upper pole of the left calyx was not successful, two ureteral stents were placed into the lower and middle pole calyces to maximize urine drainage.
After 6 weeks of conservative management with two ureteral stents, the patient continued to have profuse watery diarrhea, likely induced by continued drainage of urine into the colon.

After administration of pyridium (phenazopyridine; a urinary analgesic that is known to change the color of urine to reddish-orange), our patient's stools turned reddish-orange, confirming the presence of urine in the stool and suggesting the persistence of the colo-renal fistula. The case was reviewed by gastroenterology and endoscopic closure of the colonic fistula site was deemed to not be feasible based on the larger size of the colonic defect and its location in the splenic flexure.

Given the failure of non-operative management of the fistula, the patient was taken to the operating room and underwent an exploratory laparotomy. The fistulous connection between the splenic flexure of the colon and the upper pole of the left kidney was identified and taken down. The splenic flexure of the colon was resected and a new colo-colonic anastomosis was created. The fistula defect $(1 \times 1 \mathrm{~cm})$ in the upper 
pole of the left kidney was closed with interrupted absorbable sutures incorporating the renal parenchyma and the Gerota's fascia. A pedicled omental flap was then created and secured in place over the renorraphy site. Pathological examination of the fistula tract revealed no residual tumor. Subsequently, the patient was discharged to home and had an unremarkable recovery with resolution of his presenting symptoms. CT imaging two weeks after the surgery ruled out any extravasation, and the ureteral stents were removed uneventfully.

\section{Discussion}

Radiofrequency ablation is an increasingly utilized technique in the treatment of small, solid renal masses $(<4 \mathrm{~cm})^{6}$. During the RFA procedure, a radiofrequency probe is inserted and deployed in the ablation zone, and a generator provides an alternating current in the radiowave frequency of the electromagnetic spectrum. The tissue's impedance to this current leads to local tissue hyperthermia and results in irreversible cell injury ${ }^{6}$.

The benefits of renal RFA over surgical management of suspicious renal lesions include the possibility of avoiding general anesthesia, prevention of renal ischemic time, faster recovery, and reduced complications ${ }^{7}$.

The overall complication rate after RFA of renal tumors is $5-10 \%(9.2 \%$ minor and $1.8 \%$ major complication rate) ${ }^{8-10}$. Minor complications include skin burns, pain or paresthesia, transient hematuria, and perinephric hematomas ${ }^{3,11}$. The major complications reported in the literature include injury to the pyelo-calyceal system, gross hematuria requiring surgical exploration or angio-embolization, and bowel injury ${ }^{3,11}$.

Consideration of the anatomical location of the renal lesion is important to minimize the risk of bowel injury with RFA. Anterior tumors are more likely to be in contact with the bowel and thus increase the risk of bowel injury ${ }^{12,13}$. At least 5 $\mathrm{mm}$ of mesenteric fat should be present between the target renal tumor and the adjacent bowel, to adequately insulate the bowel from injury of the emitted radiowaves ${ }^{14}$. As such, a laparoscopic rather than percutaneous RFA is advised for anterior renal tumors in order to decrease the risk of bowel injury ${ }^{14}$. Some additional adjunctive techniques that can be used to physically separate the ablation target zone from the bowel include: positioning the patient in a decubitus position; levering the tumor away from adjacent bowel with the RFA probe; and injection of sterile fluid to hydrodissect the tumor away from the bowel ${ }^{12,13,15}$.

Colo-renal fistula formation is an extremely rare thermal complication of renal RFA, and consequently there is no data to support a consensus on the optimal treatment modality.

Medina et al. ${ }^{1}$, reported a case of colo-renal fistula after renal RFA in a patient with a left solitary kidney who had undergone two previous partial nephrectomies. The colo-renal fistula manifested 7 days following the RFA as watery diarrhea. The patient was treated with ureteral stent placement, colonic resection, and closure of the renal fistula tract. Four days after repair the patient developed a reno-peritoneal fistula and ultimately had to undergo a radical nephrectomy. Patel et al. ${ }^{2}$, diagnosed a colo-renal fistula 2 months after a patient underwent a CT-guided RFA for a left upper pole renal cell carcinoma. The patient failed one month of conservative treatment with total parenteral nutrition and ureteral stent placement, and ultimately underwent surgical resection of the colo-renal fistula (with renal preservation) and colonic resection.

Weizer et al. ${ }^{3}$, reported one fistula occurring in a patient with a left sided anterior upper pole lesion and a solitary kidney who underwent percutaneous RFA. The fistula manifested 7 days post ablation. The fistula was successfully treated conservatively with nephrostomy tube decompression and total parenteral nutrition.

Douglas et al ${ }^{4}$, published a case report of right renal RFA complicated by a delayed (10 months) colo-renal fistula that was treated with a right partial nephrectomy and right hemicolectomy. Mourmouris et al ${ }^{5}$, reported a case of post-RFA colo-renal fistula that failed conservative and 
over-the-scope colonoscopic clip placement, and ultimately required surgical intervention consisting in segmental colonic resection and suture repair of the renal defect.

As outlined above, our patient failed conservative therapy with antibiotic therapy and ureteral stenting. Therefore, a surgical intervention was performed involving segmental colonic resection and renorrhaphy, with total renal preservation.

Based on our experience with the present case, as well as on our review of previously reported cases of colo-renal fistulae after percutaneous RFA, we propose a multidisciplinary algorithm in their management in order to optimize outcomes. Clinically stable patients should first undergo a trial of non-operative management consisting of antibiotic therapy, bowel rest, nutritional support, urine drainage from the involved pyelocaliceal system, and attempt at over-thescope endoscopic closure of the colonic defect. Surgery should be reserved for cases of failed non-operative management. Complete kidney preservation is possible and every attempt to save the kidney should be made during the surgery.

\section{Conclusion}

Physicians should remain aware of the risk of colo-renal fistula following renal RFA. Earlyrecognition and a multidisciplinary approach are essential in the management of colo-renal fistulae. An attempt at non-operative management should precede surgical intervention.

\section{Lessons Learned}

Colo-renal fistulae complicating renal RFA represent a significant challenge. A multidisciplinary approach is very important in their management, with surgery being reserved for failed conservative treatment.

\section{References:}

1. Medina SJ, González RE, Hernández-Atance JM et al. Renocolic fistula as a complication of radiofrequency in the treatment of renal cell carcinoma. Arch Esp Urol. 2010 Jan-Feb;63(1):74-7.

2. Patel BJ, Mathur AK, Puri N, Jackson CS. A Rare Case of Nephrocolic Fistula Resulting from Radio
Frequency Ablation (RFA) of Renal Cell Carcinoma. ACG Case Rep J. 2014 Jan 10;1(2):93-5.

3. Weizer AZ, Raj GV, Polascik TJ, et al. Complications after percutaneous radiofrequency ablation of renal tumors. Urology. 2005 Dec;66(6):117680.

4. Douglas S, Ogles M, Vick K, Pound CR. Delayed renocolic fistula formation following percutaneous radiofrequency ablation of renal mass: case report and review of the published cases. BJU International; 10.21.2012. https://www.bjuinternational.com/case-studies/delayed-renocolic-fistulaformation-following-percutaneous-radiofreqencyablation-of-renal-mass-case-report-and-reviewof-the-published-cases/ [Accessed 11.10.2019].

5. Mourmouris $\mathrm{P}$, Mperdempes M, Papachristou C, Skolarikos $A$, et al. Renocolic fistula following radiofrequency ablation of a renal tumor. A rare case report and review of the literature. Hellenic Urology. 2018;30 (2):44-46.

6. Park S, Cadeddu JA. Outcomes of Radiofrequency Ablation for Kidney Cancer. Cancer Control. 2007; 205-210.

7. Allaf ME, Varkarakis IM, Bhayani SB, et al. Pain control requirements for percutaneous ablation of renal tumors: cryoablation versus radiofrequency ablation: initial observations. Radiology. 2005;237:366-370.

8. Park SH, Anderson JK, Matsumoto ED, et al. Radiofrequency ablation of renal tumors: intermediate-term results. J Endourol. 2006;20:569-573.

9. Arzola J, Baughman SM, Hernandez J, et al. Computed tomographyguided, resistance-based, percutaneous radiofrequency ablation of renal malignancies under conscious sedation at two years of follow-up. Urology. 2006;68:983-987.

10. Hegarty NJ, Gill IS, Desai MM, et al. Probe-ablative nephron-sparing surgery: cryoablation versus radiofrequency ablation. Urology. 2006;68 (1 Suppl):7-13.

11. Roach H, Whittlestone T, Callaway MP. Lifethreatening hematuria requiring transcatheter embolization following radiofrequency ablation of renal cell carcinoma. Cardiovasc Intervent Radiol. 2006;29:672-674.

12. Park SH, Yoon SK, Sung GT, et al. Radiofrequency ablation treatment for renal cell carcinoma: Early clinical experience. Korean J Radiol 2008; 9:340-47.

13. Gervais D, Arellano R, Mueller P. Percutaneous radiofrequency ablation of renal cell carcinoma. Eur Radiol 2005; 15: 960-67.

14. Rhim H, Dodd G 3rd, Chintapalli K, Wood B, Dupuy D, Hvizda J, Sewell P, Goldberg S. Radiofrequency thermal ablation of abdominal tumors: 
lessons learned from complications. Radiographics 2004; 24:41-52.

15. Gervais DA, Arellano RS, McGovern FJ, McDougal WS, Mueller PR. Radiofrequency ablation of renal cell carcinoma. II. Lessons learned with ablation of 100 tumors. AJR Am J Roentgenol 2005;185(1):72-80. 